\title{
Scaling Multi-Core Network Processors Without the Reordering Bottleneck
}

\author{
Alexander Shpiner \\ Technion \\ shalex@tx.technion.ac.il
}

\author{
Isaac Keslassy \\ Technion \\ isaac@ee.technion.ac.il
}

\author{
Rami Cohen \\ IBM Research \\ ramic@il.ibm.com
}

\begin{abstract}
Today, designers of network processors strive to keep the packet reception and transmission orders identical, and therefore avoid any possible out-of-order transmission. However, the development of new features in advanced network processors has resulted in increasingly parallel architectures and increasingly heterogeneous packet processing times, leading to large reordering delays.

In this paper, we introduce novel scalable scheduling algorithms for preserving flow order in parallel multi-core network processors. We show how these algorithms can reduce reordering delay while adapting to any load-balancing algorithm and keeping a low implementation complexity overhead. To do so, we use the observation that all packets in a given flow have similar processing requirements and can be described with a constant number of logical processing phases. We further define three possible knowledge frameworks of the time when a network processor learns about these logical phases, and deduce appropriate algorithms for each of these frameworks.
\end{abstract}

\section{INTRODUCTION}

\section{A. Background}

Network Processors (NPs) are specialized softwareprogrammable architectures in routers, switches and network cards. NPs are designed to process packets at high speeds, and especially to implement such diverse functions as forwarding, classification, protocol conversion, Deep packet inspection (DPI), SSL and firewalling [1]-[4].

NPs are required to avoid out-of-order transmission of the packets, because out-of-order packets can disrupt the local pipeline logic of the router, as well as significantly decrease the throughput of TCP flows [5], [6]. Unfortunately, in recent years, two trends have made it increasingly hard for NP designers to keep packets in order without suffering from a large additional delay. First, NP architectures are becoming increasingly parallel. For instance, they often rely on many parallel processing cores (e.g., the Cavium CN68XX [7] or the AMCC nP7310 [8]), or on a hybrid combination of parallel and pipeline cores (e.g., the EZChip NP-4 [9] or the Netronome NFP-32xx [10]). Second, packet processing needs are becoming increasingly heterogeneous. NPs need to implement a growing number of increasingly complex features, such as advanced VPN encryption, decompression, VoIP session border controller (SBC), video call admission control (CAC), per-subscriber queueing, and hierarchical classification for QoS [7], [11], [12].

As a consequence of these two trends of parallel architectures and heterogeneous processing delays, many packets with small processing times may be ready to leave the NP, but need to wait for a few packets that arrived earlier and are still stuck in heavy processing tasks. Therefore, these NPs exhibit high and unpredictable reordering delays, which conflict with the delay requirements that NP designers need to meet.

Current reordering algorithms typically do not handle this heterogeneous traffic gracefully. In particular, as detailed below, they either (a) cause a needless large reordering delay, or (b) rely on a static load-balancing through hashing and can cause a lower throughput.

In this paper, our goal is to provide a scalable algorithm that reduces the reordering delay, while adapting to any loadbalancing scheme. Thus, the NP designer can keep using the same load-balancing scheme that achieves high throughput, and just apply our algorithm to reduce the reordering delay.

We introduce a new model for NPs. In particular, we make the observation that all the packets of a given flow can typically be divided into an equal number of well-defined logical processing phases, which correspond to their similar processing requirements.

Next, we introduce three algorithms, called Reordering Per Processing Phase $\left(R P^{3}\right)$, which leverage this knowledge to reduce the reordering delay. We further illustrate how these algorithms are implemented.

Finally, using extensive simulations based on both synthetic traffic and real-life traces, we analyze our $R P^{3}$ algorithms and show that their reordering delays are negligible when compared to previously known techniques. We also illustrate how a lower variability in the delays of the logical processing phases leads to significant improvements in the reordering delays of our algorithms.

Our online technical report [13] presents additional technical details, including the algorithm under Framework 2, the proofs of correctness of the various algorithms, a discussion of the implementation complexity, and extended simulations.

\section{B. Related Work}

Recent research works have described several architectures that aim to reduce the reordering delay. First, pipeline-based architectures without parallelism clearly preserve the packet order. However, they are hardly scalable, because of the heterogenous requirements of the packets, the synchronization requirements, and the granularity of the processing commands [14]. Thus, we further discuss only parallel architectures.

Statically mapping each flow to a single core using hashing is another popular way to intrinsically avoid reordering [15]. However, it results in an insufficient utilization of the cores, and therefore in a lower throughput, due to the fact that several elephant flows may map to the same core [16]. Moreover, it is possible to adapt the load-balancing scheme by using feedback 
on the core utilization in order to increase throughput [17], [18]. However, this can also cause packet reordering [19], and therefore requires an ordering mechanism. In addition, all these approaches fix the load-balancing scheme, while we would like to adapt to any potential scheme.

There are several algorithms for keeping packet order without changing the load-balancing scheme. First, the NP can allocate a global sequence number to every arriving packet [20], [21]. However, this solution also incurs a high reordering delay, because all packets are treated as a single flow, the order of which has to be preserved. A second, ideal approach is to rely on per-flow sequencing [21]-[23], thus providing a minimal reordering delay. However, as explained in [16], it is not scalable to a large number of network flows because of the large number of needed counters. A third method to avoid packet reordering is to keep an inter-thread signaling system between the cores [20]. However, in NPs with a high degree of parallelism and a high clock frequency, this method can be complex to implement.

A fourth and last appealing approach is to statically aggregate flows by hashing the flow identifier in the packet header into several ordering domains [16]. A different sequence number generator is then assigned for each ordering number. The flow identifier may for instance consist of its 5-tuple and its input interface. In each of the ordering domains, the order of the packets is preserved. However, an unnecessary reordering delay occurs between flows with different processing requirements that are hashed into the same ordering domain. In particular, this method suffers from the fact that flows in the same domain do not necessarily have similar processing delays. By contrast, we suggest to base reordering domains on the processing phases, thus following natural flow properties rather than using arbitrary hashing. Note that the two solutions can also be combined, by simultaneously distinguishing flows based on an arbitrary hash as well as on the number of processing phases.

\section{System Model AND PROBLEM ForMUlation}

\section{A. System Model}

Consider a network processor (NP) with $N$ processing elements (PEs), which can also be general-purpose CPU cores. We allow for any load-balancing scheme. Therefore, each arriving packet can be allocated to any arbitrary PE for processing. As commonly assumed, we consider a single stream of incoming packets [16], [22]-[25]. We allow arbitrary arrival times of the packets, and assume infinite buffer sizes. Also, we neglect the different priority levels of the flows. The management of the packets in the NP is performed with packet descriptors, which hold all the necessary information for packet processing, including pointers to the packets in the main buffer.

We mandate that packets from the same flow depart in the order of their arrival. When a packet needs to wait for another packet to make sure that it departs in order, it experiences a reordering delay. Our goal is to reduce the average reordering delay, using a simple and scalable algorithm.

\section{B. Assumptions}

The main assumption in this paper is that the processing requirements of all the packets from the same flow can be divided into an equal number of logical processing periods, denoted as phases (as in [24]). For instance, all the packets of a given flow may only require forwarding, while all the packets of another flow may need forwarding, deep packet inspection, and IPSec processing.
This requirement is reasonable in practice because phases are only logical, and we do not mandate that all phases correspond to the same number of cycles. For instance, if the first and last packets in a flow require a higher number of phases because the network processor needs to open or close flow-based states, then we can simply insert some empty dummy logical phases in the other packets. Of course, a higher variability of the actual time of each phase will adversely impact the reordering delay of any algorithm based on this assumption. We also allow large processing functions such as deep packet inspection to be further subdivided into different logical phases, as long as all the packets of a given flow experience the same subdivision. Finally, we denote by $\Phi \in \mathbb{N}$ the maximal possible number of processing phases per packet.

In practice, the required number of processing phases can be obtained after parsing the packet header, and comparing its content to the user-configured rules for specific flows or protocols. Therefore, the NP does not need to store the total number of phases for each of the flows. For example, if the packet includes an IPSec header, the configuration table may indicate that the packet needs to be authenticated and forwarded. Further, if an authentication needs ten processing phases and IP forwarding needs one processing phase, the NP deduces that the packet requires eleven processing phases.

In addition, if the processing cannot be subdivided into logical phases for some specific flows, then it is still possible to treat those flows as in current NPs. In particular, those flows can be sent to other ordering domains by hashing the packet header as in [16]. This type of ordering domain can coexist in parallel with our processing-based reordering domains.

\section{Knowledge Frameworks}

Estimating the processing time of each packet can significantly help in reducing the average reordering delay. Nevertheless, in many cases such an estimation can only be realized during, or at the end of the data processing.

In this section, we define three independent knowledge frameworks regarding the time at which the NP knows about the number of processing phases of each packet. In our discussions with industry NP designers, we found that these frameworks capture different assumptions and capabilities of NP vendors and architectures. Our goal is to study the impact of each knowledge framework on the complexity of the reordering algorithm and on the resulting reordering delay. The following three frameworks are ordered from the framework with more knowledge to the framework with less knowledge, and therefore are expected to result in an increasingly high reordering delay. This is confirmed in simulations (Section VI).

The first framework assumes that there is a header parser before load-balancing, thus ensuring a full knowledge of the number of the phases of each packet before it is load-balanced among the PEs. This framework is the simplest one to analyze, and it conveys some intuition on the problems involved. It is often adopted in the literature [24]-[29].

Framework 1: The number of processing phases of a packet is known upon its arrival at the network processor.

In many cases, once a packet arrives at a PE, its first processing phase includes packet classification, based on the packet headers (including in some cases the application header) [30]. This packet classification can help us in determining the number of remaining processing phases, leading to a second 
framework.

Framework 2: The total number of processing phases of a packet is only known after its first processing phase.

In most cases, we would expect the total number of phases to be known after the first processing phase. However, in cases where the processing of the packet is performed recursively without a predetermined number of iterations, as in MPLS or PBB label encapsulation [31], it is hard to know the number of processing phases in advance [32]. More generally, we can introduce a third framework, in which we only know the number of processing phases a packet has already gone through, but not the number of remaining ones.

Framework 3: The PE only knows about the number of processing phases a packet has already gone through, but not about the number of remaining ones. Therefore, the total number of processing phases of a packet is known only after its processing is completed.

\section{III. $R P^{3}$ AlgORITHM UNDER FRAMEWORK 1}

In this section, we introduce our order-preserving $R P^{3}$ (Reordering Per Processing Phase) algorithm for Framework 1, where the number of phases of each packet is known upon packet arrival. Since Framework 1 provides full knowledge of the number of phases, the resulting algorithm is relatively simple, and will provide some intuition on the mechanism before describing more complex algorithms under later frameworks with limited knowledge.

Intuitively, the algorithm divides all the flows into subsets of flows that require the same number of processing phases. The goal is to avoid a case in which a packet belonging to a flow with many processing phases blocks a packet belonging to a flow with few processing phases at the reordering unit, as illustrated in the Introduction. By reducing the amount of such blocking, the algorithm decreases the reordering delay.

Figure 1 presents the $R P^{3}$ algorithm under Framework 1. The algorithm relies on an architecture that includes $\Phi$ sequencenumber (SN) generators, and $\Phi$ ordering units (OUs). The $\phi$-th $\mathrm{SN}$ generator assigns the next SN for packets with $\phi$ processing phases. The $\phi$-th ordering unit tracks the latest released packet with $\phi$ processing phases. Each ordering unit has $N$ input buffers, one per PE. Note that while the ordering units are presented as separate for intuition, in practice they may be implemented on a single core.

First, each packet is immediately assigned an SN upon arrival, as shown in step (1) of Figure 1. In Framework 1, the number of required processing phases $\phi$ of the packet is known as soon as it arrives. Therefore, the $\mathrm{SN}$ of the packet is assigned by the $\phi$-th SN generator (step (2) in Figure 1), which also increments its last assigned SN.

Next, the packet descriptor is sent by the load balancer to some PE $i$. After finishing the processing in PE $i$, the packet descriptor is placed in buffer $i$ of ordering unit $\phi$ (step (3)). The ordering unit can only release in-order packets. To do so, each ordering unit $\phi$ checks if one of the $N$ head-of-line packets in its buffers has an $\mathrm{SN}$ equal to the next expected $\mathrm{SN}$, i.e. to the $\mathrm{SN}$ of the oldest packet in the NP with $\phi$ phases (step (4)). If the condition is met, the packet can depart, and the expected SN is incremented (step (5)). Else, the packets keep waiting for the next expected packet.

Each ordering unit $\phi$ preserves the order of the packets that require $\phi$ processing phases. Thus, reordering delay can only

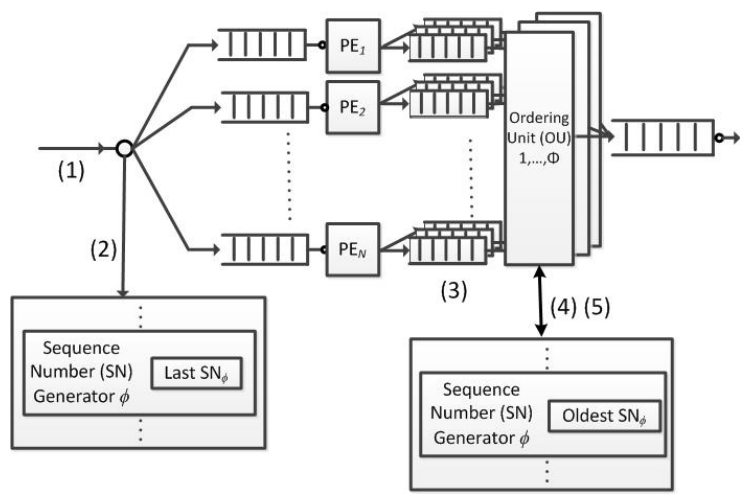

Fig. 1. $R P^{3}$ Algorithm under Framework 1. Packets with a different number of phases belong to different flows. Thus, they will not affect the reordering delay of each other.

occur within those packets. For instance, assume that packets $A$ and $B$ belong to two different flows with the same number of logical processing phases, and $A$ arrives to the NP before $B$, but $B$ has completed its processing earlier. Then $B$ will wait for $A$ in order to depart, even though they belong to different flows, because they have the same number of phases. As we will show in simulations (Section VI), this reordering algorithm achieves a significantly low reordering delay, and this is especially true when the variability in the delay of each processing phase is low.

The $R P^{3}$ algorithm under Framework 2 is quite similar to the described $R P^{3}$ algorithm under Framework 1, and therefore we detail it in our online technical report [13].

\section{IV. $R P^{3}$ Algorithm UNDER FramewOrK 3}

\section{A. Overview}

Under Framework 3, the PEs only know the number of processing phases a packet has already gone through, but not the number of remaining ones.

Intuitively, the problem in Framework 3 is that a packet that has completed its processing by going through $\phi$ processing phases is blocked from departing the NP by all the packets that have arrived earlier, have completed less than $\phi$ processing phases, and are still in some PE. This is because all these other packets may, or may not, eventually complete with exactly $\phi$ processing phases, and therefore potentially belong to the same flow. So the algorithm needs to monitor all these other packets to determine whether the packet is free to go. This intrinsically introduces three new problems in the $R P^{3}$ algorithm:

First, each packet now needs to go through several sequencenumber (SN) generators, since it doesn't know its SN generator in advance. For instance, assume a packet needs to complete $\phi$ processing phases. Since it doesn't know it before completion, it will first ask for the first SN generator; then, for the second SN generator, and so on, gradually discovering how many phases it has.

A second new problem is that when a packet requests a new SN, it cannot get it automatically anymore. Consider the following example, which we later extend in Figure 3. Assume that incoming packet $\mathrm{A}$ is assigned $S N=1$ by the first $\mathrm{SN}$ generator, and the next incoming packet B is assigned $S N=2$ by the same first SN generator. Now, assume that packet B completes its first processing phase, but packet A hasn't yet. If $\mathrm{B}$ needs to go through a second processing phase and requests an SN from the second SN generator, which one should it get? 
We do not know yet whether A will also request an $\mathrm{SN}$ from the second SN generator, and therefore do not know if the second $\mathrm{SN}$ for B should equal 1 or 2 . Therefore, A blocks the SNgranting process for B. More generally, the algorithm preserves flow order by making sure to only increase the SN of the oldest packet in the current sequence phase.

A third new problem is that we want to design our algorithm so that each PE is work-conserving, i.e. each packet on each PE can run-to-completion independently of the sequencing scheme. As a consequence, the packet processing continues even if the new $\mathrm{SN}$ is not granted yet. This makes the processing phases and sequencing phases distinct. For instance, a packet may be in the middle of the processing of phase 4 , but its sequence number may still belong to phase 2 . While this makes the algorithm more efficient, it also makes it significantly more complex to understand.

We further denote as a sequence phase the time between receiving two consecutive $\mathrm{SN}$ grants. Note that the final number of sequence phases for a packet is equal to its total number of required processing phases.

Let's now formally describe the algorithm before providing a clarifying example.

\section{B. Algorithm Description}

Figure 2 illustrates the $R P^{3}$ Algorithm under Framework 3. Assume that the maximum possible number of processing phases per packet is $\Phi$. The architecture includes $\Phi$ SN generators, and $\Phi$ ordering units (OUs) with $N+1$ input queues, numbered 0 through $N$. The $\phi$-th $\mathrm{SN}$ generator is responsible for preserving the order of all the packets that are currently in the $\phi$-th sequence phase. The $\phi$-th ordering unit holds the packets that have finished processing and are currently within the $\phi$-th sequence phase. A packet sequence number is defined by the pair $\left(\phi: S N_{\phi}\right)$, where the SN generator that assigns the sequence number is the $\phi$-th $\mathrm{SN}$, and the sequence number that it assigns is $S N_{\phi}$.

Upon arrival, a packet is assigned a sequence number $S N_{1}$ by the $\mathrm{SN}$ generator 1 (step (1) in Figure 2). It then joins the queue of one of the PEs, as determined by the load-balancer.

After finishing the $\phi$-th processing phase of the packet in the $\mathrm{PE}$, a request token with $S N_{\phi}$ is sent to $\mathrm{SN}$ generator $\phi$ (step (2) in Figure 2). The SN generator $\phi$ checks if the sequence number $S N_{\phi}$ of the request token is the minimal one, i.e. the oldest sequence number of the packets in sequence phase $\phi$. When the condition is met, $S N_{\phi+1}$ is retrieved from SN generator $\phi+1$ and $S N_{\phi}$ is released. A grant token with $S N_{\phi+1}$ is sent, and the sequence number of the packet is updated (step (3)).

This completes a single sequence phase. When PE $i$ finishes processing the packet, the packet descriptor is sent to the input queue $i$ of the ordering unit $\phi$, where $\phi$ is the number of passed packet sequence phases (step (4)).

At this point, as mentioned, the current number of sequence phases of the packet, i.e. the number of SN re-assignments of the packet, may be smaller than the total number of processing phases it needs to achieve. This is because the packet is waiting for some SN grant token, due to some earlier-arrived packet that has not yet completed processing. As we stated before, the final number of sequence phases has to be equal to the total number of processing phases. Thus, the packet will complete the needed sequence phases in the ordering unit. (Such a case is further illustrated in the example below with packet $D$.)

For each of the head-of-line packets in its input queues,

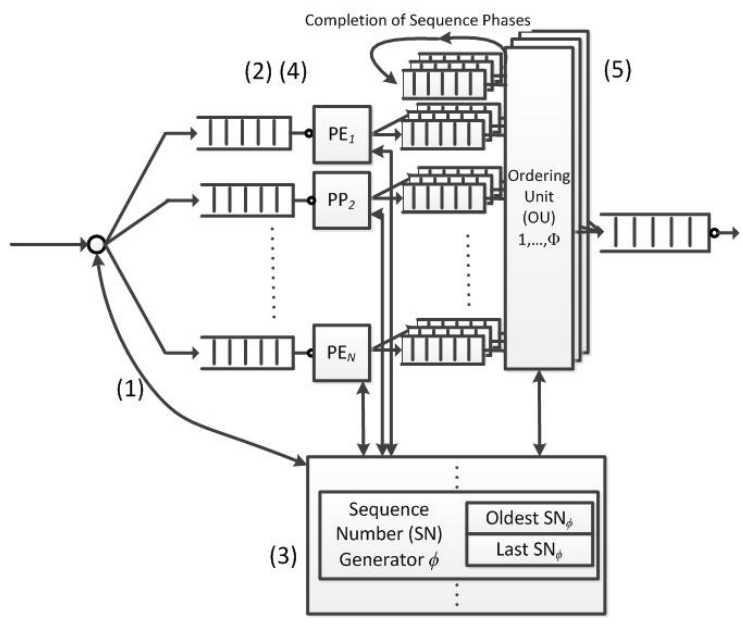

Fig. 2. $R P^{3}$ Algorithm under Framework 3. Each packet with $\phi$ required processing phases also passes through $\phi$ sequencing phases. In each sequencing phase, a request token is sent to the SN generator, and a grant token is received. The SN generator sends a grant token only when the requested $\mathrm{SN}$ is equal to the oldest SN counter. The generated grant token includes the next SN from the next SN generator. The packet order is preserved for the packets in the same sequencing phase.

ordering unit $\phi$ will send a request token to $\mathrm{SN}$ generator $\phi$. After receiving the grant token $S N_{\phi+1}$, the packet is pushed to the input queue numbered 0 of ordering unit $\phi+1$. More generally, input queue 0 is reserved for packets that completed their sequence phase in another ordering unit, while input queues 1 to $N$ are reserved for the packets that were pushed from the PEs.

Finally, when the number of passed sequence phases is equal to the total number of processing phases, the packet departs and its sequence number is released (step (5)).

The next example illustrates and clarifies how the $R P^{3}$ algorithm works under Framework 3.

Example 1: Figure 3 shows an example of sequence number assignment and the in-order transmission of the packets. Packets $\mathrm{A}, \mathrm{B}, \mathrm{C}$ and $\mathrm{D}$ arrive to the NP in this order, and are processed in parallel. Packets $\mathrm{A}$ and $\mathrm{C}$ require one processing phase $\left(\phi_{A}=\phi_{C}=1\right)$, and may belong to the same flow. Packet $\mathrm{B}$ requires three processing phases $\left(\phi_{B}=3\right)$, and therefore belongs to a different flow. Packet $\mathrm{D}$ requires two processing phases $\left(\phi_{D}=2\right)$, and belongs to yet another flow. Of course, in Framework 3, the number of processing phases required by each packet is unknown to the NP until packet processing is completed. The rectangles in the figure present the completion time of each processing phase. For instance, $t_{A, 1}, t_{B, 1}$ and $t_{C, 1}$ are the completion times of the first processing phase of packets $\mathrm{A}, \mathrm{B}$ and $\mathrm{C}$, respectively.

Notice first that the first increment of the sequence number of packet $\mathrm{B}$ happens only after incrementing the sequence number of packet $\mathrm{A}\left(t_{A, 1}\right)$, and not immediately after finishing its first processing phase $\left(t_{B, 1}\right)$. This is because the $\mathrm{SN}$ generator cannot assign the next $\mathrm{SN}$ to packet $\mathrm{B}$ as long as it does not know that packet A will not need it.

In addition, once the processing of packet $\mathrm{C}$ completes after one processing phase, it still needs to wait for $t_{A, 1}$ and $t_{B, 1}$ in order to leave the NP. The reason is that as long as A and B do not complete their first phase, the NP does not know whether A and $\mathrm{B}$ need only one processing phase, in which case they may 


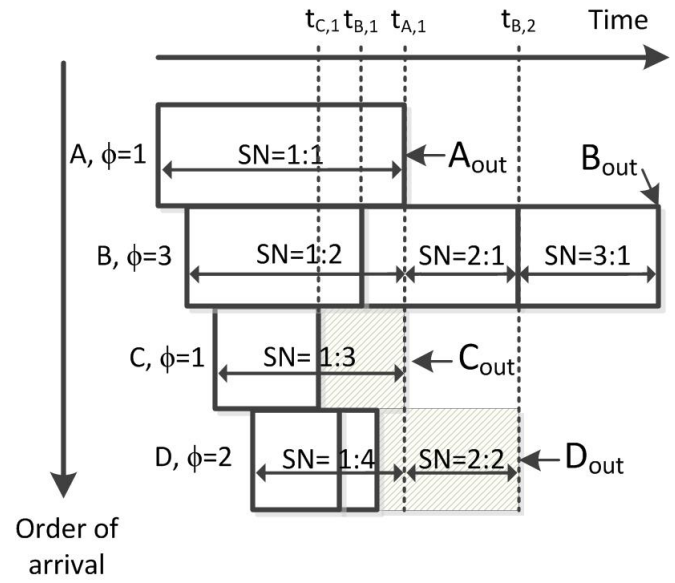

Fig. 3. Sequence Numbering Example. Rectangles present the processing phases, and double arrows illustrate the SN generators and values. A packet sequence number is defined by the pair $\left(\phi: S N_{\phi}\right)$, where the $\mathrm{SN}$ generator that assigns the sequence number is the $\phi^{\text {th }} \mathrm{SN}$, and the sequence number that it assigns is $S N_{\phi}$. Packets A, B, C and D are processed in parallel. Packet C can be transmitted only after $\mathrm{A}$ and $\mathrm{B}$ complete their first sequencing phase at $t_{A, 1}$ and $t_{B, 1}$. The reordering delay in this case is equal to $t_{A, 1}-t_{C, 1}$. Packet $\mathrm{D}$ completed its processing phases before completing its sequencing phases, and waits for $\mathrm{B}$ to complete its second sequencing phase at $t_{B, 2}$.

belong to the same flow, or more processing phases, in which case they definitely do not belong to the same flow. Of course, packet $\mathrm{C}$ does not need to wait for $t_{D, 1}$, because packet $\mathrm{D}$ arrived after packet $\mathrm{C}$.

In this case, the reordering delay of packet $\mathrm{C}$ is equal to $t_{A, 1}-t_{C, 1}$. Note that with previously-known reordering algorithms, the reordering delay of $\mathrm{C}$ could have been larger and equal to $t_{B, 3}-t_{C, 1}$, since $\mathrm{C}$ would have waited for the processing completion of $\mathrm{B}$. Also note that packet processing is never preempted, even if the next sequence number cannot be received, i.e. the suggested algorithm does not pause packet processing in any case.

Finally, packet D finishes both its processing phases before the increment of its sequence number, and is buffered in the ordering unit. It can be transmitted only after packet $\mathrm{B}$ finishes its second sequencing phase at time $t_{B, 2}$.

As mentioned, in our online technical report [13], we further discuss the implementation overhead and prove the correctness of the $R P^{3}$ algorithm. Specifically, we estimate the addional control overhead as 40, 60 and 160 bits per packet in Framework 1,2 and 3 , respectively.

\section{PERFormance AnAlysis Model}

To provide more intuition on the efficiency of our $R P^{3}$ algorithm, we now analyze its reordering delay and total delay as functions of the traffic arrival pattern and the processing delay distribution. Specifically, we want to compare the $R P^{3}$ algorithm against two baseline architectures:

Single SN algorithm: A commonly-implemented and straightforward way to preserve packet order is to use a single global sequence number ( $\mathrm{SN}$ ) generator. Each arriving packet is simply stamped with an incremented sequence number. Then, the reordering unit at the output link transmits only packets with the oldest sequence number. Other packets keep waiting. We will also later show that while this simple architecture is easy to implement, it can cause high reordering delays, because packets of one flow may need to wait for a long time for late packets of a different flow.

Hashed SN algorithm [16]: As discussed in the related work, it is also possible to statically aggregate flows into ordering domains using hashing. We will use this algorithm as our second baseline algorithm, and denote it as Hashed $S N$.

We start by defining the potential reordering delay of a packet as the difference between its arrival time, and the latest departure time of a previously-arrived packet that requires order preservation. For example, consider two packets $A$ and $B$, such that their order needs to be preserved. Assume that $B$ arrives at $t_{\text {arr,B }}$, and $A$ departs at $t_{\mathrm{dep}, \mathrm{A}}$. Then the potential reordering delay of packet $B$ is defined as $\max \left(t_{\mathrm{dep}, \mathrm{A}}-t_{\mathrm{arr}, \mathrm{B}}, 0\right)$.

Our goal is to present the cumulative distribution function of the total delay $T_{T}$, given the distributions of the processing delay $\left(T_{\text {proc }}\right)$ and of the potential reordering delay $\left(T_{\mathrm{RO}}\right)$. We make several simplifying assumptions. First, we assume that the processing delay includes both the processing time and the buffering time. We also assume that we can neglect the transmission delay of the packets at the output. We further assume that the processing delay and the potential reordering delay follow independent distributions, and that the processing delays of different packets are independent as well. Finally, we assume a common slotted-time model [33].

The following lemma first describes a general delay model that will be used in the later theorems.

Lemma 1: The distribution of the total packet delay can be modeled as:

$$
\operatorname{Pr}\left(T_{T} \leq i\right)=\operatorname{Pr}\left(T_{\text {proc }} \leq i\right) \cdot \operatorname{Pr}\left(T_{\mathrm{RO}} \leq i\right),
$$

where $\operatorname{Pr}\left(T_{\mathrm{RO}} \leq i\right)$ depends on the arrival traffic pattern and on the scheduling algorithm.

Proof: A packet $B$ that completes processing at some time $t$ can depart iff there is no earlier packet $A$ preventing it from leaving at $t$; and if there is such a packet, it can only depart when the last such bottleneck-packet $A$ departs. In other words, the actual departure time of $B$ will be $\max \left(t_{\mathrm{dep}, \mathrm{A}}, t_{\mathrm{dep}, \mathrm{B}}\right)$ (where $t_{\mathrm{dep}, \mathrm{B}}$ is the depature time of $B$ when no reordering delay occurs, i.e. end of processing of $B$ ). As mentioned, we neglect the transmission delay of packet $B$ at the output. Therefore, its total time in the NP will be

$$
\begin{aligned}
T_{T} & =\max \left(t_{\mathrm{dep}, \mathrm{A}}, t_{\mathrm{dep}, \mathrm{B}}\right)-t_{\mathrm{arr}, \mathrm{B}} \\
& =\max \left(\max \left(t_{\mathrm{dep}, \mathrm{A}}-t_{\mathrm{arr}, \mathrm{B}}, 0\right), T_{\mathrm{proc}}\right) \\
& =\max \left(T_{\mathrm{RO}}, T_{\mathrm{proc}}\right),
\end{aligned}
$$

where the second max in the second line is of course superfluous. Therefore, the independence of the random variables $T_{\mathrm{RO}}$ and $T_{\text {proc }}$ yields the result.

The next theorems model the behavior of the algorithms under Poisson arrivals of rate $\lambda$. We first analyze the behavior of the Hashed SN algorithm, with $m$ buckets in the hash table. The behavior of the Single SN algorithm is easily derived using $m=1$.

Theorem 1: Under Poisson-distributed traffic arrivals with total arrival rate of $\lambda$ packets per time-slot, the total packet delay distribution under the Hashed SN reordering algorithm satisfies:

$\operatorname{Pr}\left(T_{T} \leq i\right)=\operatorname{Pr}\left(T_{\text {proc }} \leq i\right) \cdot \exp \left(-\frac{\lambda}{m} \sum_{j=1}^{\infty}\left(\operatorname{Pr}\left(T_{\text {proc }}>i+j\right)\right)\right)$. 
Proof: Following Lemma 1, we only need to focus on the second part of the right-hand side, i.e. on the reordering time. Consider some packet $B$ that arrives at time $t$, and let's find the probability that it is not blocked by an earlier packet beyond time $t+i$ due to reordering. First, at the earlier slot $(t-j)$, the probability that exactly $k$ packets arrive to the same bucket is $f_{P}\left(\frac{\lambda}{m}, k\right)$, according to the Poisson distribution. The processing of each of these packets is delayed beyond $t+i$ and causes potential reordering delay with probability $\left(1-\operatorname{Pr}\left(T_{\text {proc }} \leq i+j\right)\right)$, since $(t+i)-(t-j)=i+j$ time slots need to pass. The result follows by multiplying all the probabilities that there is no such late blocking packet from slot $(t-j)$ over all such possible slots:

$$
\begin{aligned}
\operatorname{Pr}\left(T_{T} \leq i\right) & =\operatorname{Pr}\left(T_{\text {proc }} \leq i\right) . \\
& \prod_{j=1}^{\infty}\left(1-\sum_{k=1}^{\infty} f_{P}\left(\frac{\lambda}{m}, k\right)\left(1-\left(\operatorname{Pr}\left(T_{\text {proc }} \leq i+j\right)\right)^{k}\right)\right),
\end{aligned}
$$

where $f_{P}(\lambda, k)=\lambda^{k} e^{-\lambda} / k$ ! is the Poisson-distributed probability for the arrival of $k$ packets in a time slot. Using Taylor series function $e^{x}=\sum_{n=0}^{\infty} \frac{x^{n}}{n !}$ we get the result. Ref. [13] includes the detailed proof of the theorem.

We now introduce models of the total delay in the $R P^{3}$ algorithm under Frameworks 1 and 3 (the detailed proofs are in [13]). To do so, we denote $T_{\text {proc }}^{\prime}(\phi)$ as the sum of the processing delays of the first $\phi$ processing phases of a packet.

Theorem 2: Under a Poisson-distributed traffic arrivals with total arrival rate of $\lambda$ packets per time-slot, the distribution of the total packet delay with the $R P^{3}$ reordering algorithm under Framework 1 can be modeled as:

$$
\begin{gathered}
\operatorname{Pr}\left(T_{T} \leq i\right)=\sum_{\phi_{0}} \operatorname{Pr}\left(\phi=\phi_{0}\right) \cdot \operatorname{Pr}\left(T_{\text {proc }}^{\prime}\left(\phi_{0}\right) \leq i\right) \\
\quad \exp \left(-\lambda \sum_{j=1}^{\infty} \operatorname{Pr}\left(T_{\text {proc }}^{\prime}\left(\phi_{0}\right)>i+j\right)\right)
\end{gathered}
$$

Theorem 3: Under Poisson-distributed traffic arrivals with a total arrival rate of $\lambda$ packets per time-slot, the total packet delay distribution under $R P^{3}$ for Framework 3 satisfies:

$$
\begin{aligned}
& \operatorname{Pr}\left(T_{T} \leq i\right)=\sum_{\phi_{0}} \operatorname{Pr}\left(\phi=\phi_{0}\right) \cdot \operatorname{Pr}\left(T_{\text {proc }}^{\prime}\left(\phi_{0}\right) \leq i\right) . \\
& e^{-\lambda \sum_{j=1}^{\infty}\left(1-\sum_{\phi^{\prime}=1}^{\infty} \operatorname{Pr}\left(\phi_{j}=\phi^{\prime}\right) \cdot \operatorname{Pr}\left(T_{\text {proc }}^{\prime}\left(\min \left(\phi_{0}, \phi^{\prime}\right)\right) \leq i+j\right)\right) .}
\end{aligned}
$$

Incidentally, note that the presented expressions in this section contain infinite products, but all of them converge in practice. In particular, the Poisson probabilities decrease exponentially fast for large $k$, and the number of phases is typically bounded.

\section{Simulations}

\section{A. Simulation Settings}

To evaluate our suggested algorithms, we simulate a parallel network processor with $N=16$ cores. The NP provides a pull-based I/O interface in which incoming packets are stored in a shared input queue serving all the PEs, thus achieving an efficient load-balancing. Related architectures are reported to be implemented in the Cisco QuantumFlow [11] and EZChip NP-4 [9].
We implement our $R P^{3}$ algorithm under the three frameworks. We further compare it against our two baseline algorithms, Single SN and Hashed SN. We also compute a reordering delay lower-bound, achieved using an idealized algorithm that would keep a per-flow sequence numbering mechanism. Time is continuous, and reordering delay is measured in time units.

To analyze the performance of our algorithms, we start by using a synthetic traffic arrival pattern. We assume that packet arrivals follow a Poisson distribution. Packets are distributed across 300 flows. The distribution of the flows is assumed to follow a power law (Zipf-distributed with exponent $s=1$ ) [34]. For each flow, the number of logical processing phases for all of its packets is chosen uniformly over the $[1,10]$ interval. Moreover, for a fair comparison, when implementing the Hashed-SN algorithm, the flows are hashed into 10 hash buckets. Note that we use a real hash function implementation [35], and not simply a random number generator.

Next we want to verify the impact of the delay variability of the logical processing phases on the performance of our $R P^{3}$ algorithms. Intuitively, we would expect our algorithms to perform better when delay variability is low. We add to our simulations a lower bound delay graph, which is the reordering delay obtained by a perfect reordering algorithm in which a packet can endure a reordering delay due to other packet of the same flow only. In our simulations, phase delay variability model, while keeping the mean processing time for each phase at 100 time units. In this model, we assume that the processing time for each phase is uniformly distributed over some interval. Specifically, we define the phase processing delay variability as the ratio between the maximal and minimal processing time for a single phase. Each processing phase delay is then uniformly distributed between the minimum and the maximum values, with an average of 100 time units. For instance, a phase processing delay variability of 3 corresponds to a factor of 3 between the minimum and the maximum, i.e. the minimum is 50 and the maximum is 150 . A packet with two phases would consecutively draw two such uniformly-distributed random variables.

\section{B. Traffic Load}

Figure 4 illustrates the impact of traffic load on reordering delay under the two variability models. First, we use a phase variability model with phase processing delay variability of 2. As shown, the proposed $R P^{3}$ algorithms outperform both baseline algorithms. In particular, the $R P^{3}$ algorithms reduce the reordering delay by at least an order of magnitude compared to Hashed SN. Note that the results for $R P^{3}$ for Framework 1 and Framework 2 appear near-identical throughout the simulations, and therefore they are presented as a unique algorithm. Finally, the lower bound gets positive values due to the phase processing variability.

\section{Phase Processing Delay Variability}

We then check the performance of the $R P^{3}$ algorithms under increasing processing delay variability, using the phase variability model . As shown in Figure 5, we vary the phase processing delay variability value from $1 x$ to $10 x$ under a traffic load of $90 \%$. As mentioned before, the correctness of our proposed algorithms is not affected by these variations. However, it is clear that their relative performance is reduced as the variability increases. Still, even with a 10x variability and the unfavourable Framework 3, our $R P^{3}$ algorithm interestingly keeps yielding a better result than the two baseline algorithms. 


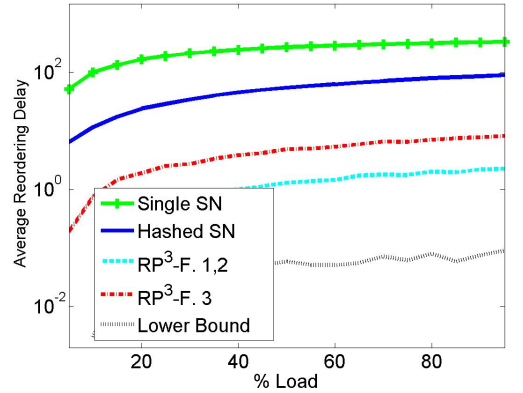

Fig. 4. Impact of load on reordering delay assuming a phase delay variability of 2 , i.e. a phase delay in $(67,133)$.

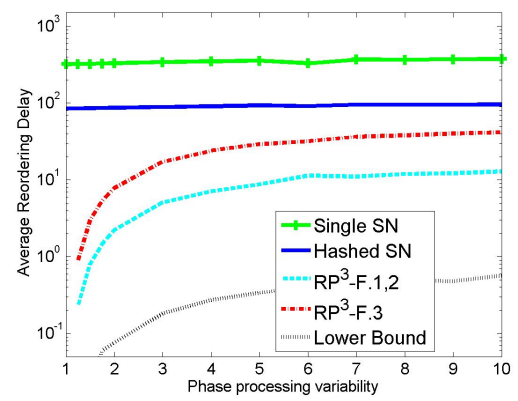

Fig. 5. Impact of delay variability under a load of $90 \%$ in the phase variability model.

\section{Real-life Trace Simulations}

We next run a set of simulations using a real-life traffic trace from CAIDA [36]. In order to evaluate the processing delay for each packet we assume that each packet is being processed for IP forwarding. We use results from Figure 9 in [37] in order to predict the processing delay of the IP forwarding as a function of packet length. We extrapolate the delay measurements presented in [37] as:

$$
\text { Delay }[\mu s]=0.266 \cdot \text { length }[\text { bytes }]+200
$$

We use the two variability models. In the phase variability model, the PE variation is chosen according to Figures 3(a), (c) and (e) in [37] as approximately equal to $1.22 \mathrm{x}$.

Note that in the trace the lengths of all the packets of the same flow are approximately equal, and therefore by Equation (5) also their processing delays. Thus, when grouping packets by their processing delays, the packets of given flows naturally belonged to the same ordering domains in the simulation, without any need for adding dummy phases. This nicely matched our assumptions without any need for additional tweaks.

Figure 6 presents the simulation results for the average reordering delay as a function of the load, under a phase variability model. In all simulations our $R P^{3}$ algorithm outperforms the compared Single-SN and Hashed-SN algorithms. The similarity between all the plots in spite of the different models suggests a relative robustness of our algorithms.

\section{E. Model Evaluation}

We also run simulations in order to evaluate our models. The models are checked by simulations with Poisson traffic of arrival rate $\lambda=1$. The number of phases per packet is assumed to be distributed uniformly and geometrically (with $p=0.5$ ) between

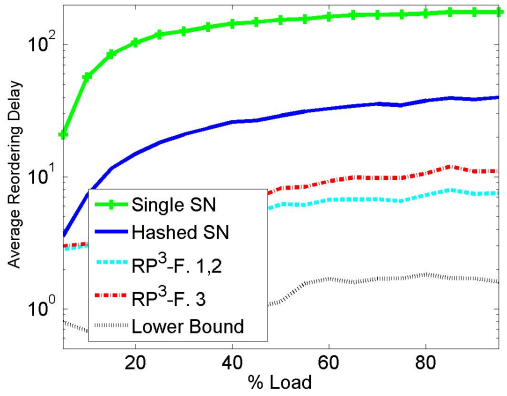

Fig. 6. Real-life trace: Impact of load on reordering delay assuming a phase delay variability of 2 , i.e. a phase delay in $(67,133))$

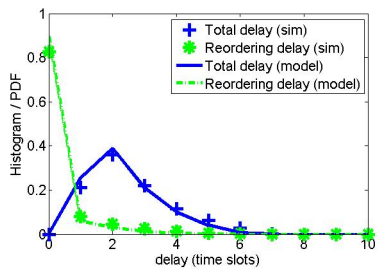

(a) Poisson arrivals, geometric processing delay distribution.

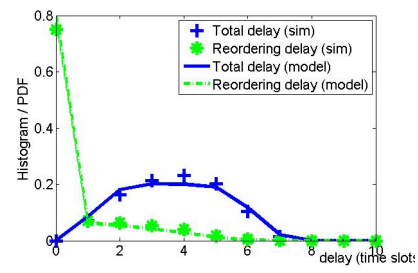

(b) Poisson arrivals, uniform processing delay distribution.
Fig. 7. Model vs. Simulation. PDF/histogram of the delay under Hashed SN

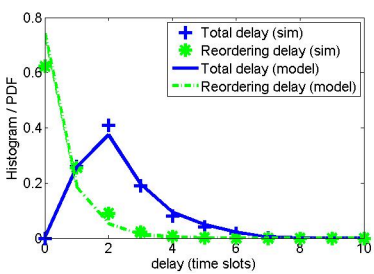

(a) Poisson arrivals, geometric processing delay distribution.

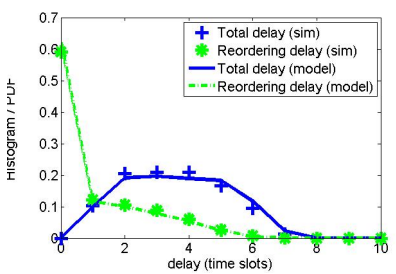

(b) Poisson arrivals, uniform processing delay distribution.
Fig. 8. Model vs. Simulation. PDF/histogram of the delay under $R P^{3}$ for Framework 1

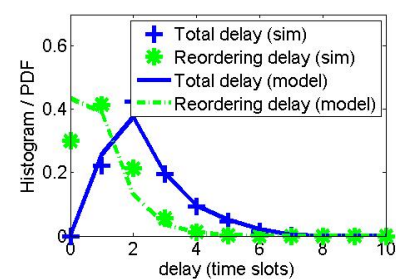

(a) Poisson arrivals, geometric processing delay distribution.

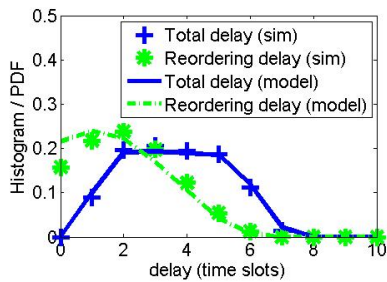

(b) Poisson arrivals, uniform processing delay distribution.
Fig. 9. Model vs. Simulation. PDF/histogram of the delay under $R P^{3}$ for Framework 3

1 and 5 . In the Hashed $\mathrm{SN}$ and the $R P^{3}$ algorithms, the flows are distributed among 5 buckets. We validate our models given a phase variability model. We set the phase processing variability to a factor of $3 \mathrm{x}$ (phase delay in $(50,150)$ ).

Figures 7, 8 and 9 compare the model and the simulation results for the Single SN, Hashed SN, $R P^{3}$ for Framework 1 and $R P^{3}$ for Framework 3 algorithms, respectively. The model fits simulations well in all cases. The simulations present some 
difference with the model due to the fact that in the simulations the arrivals use a continuous time, while the model relies on a discrete-time approximation, as well as due to the model assumptions. Note that the buffering delay appeared negligible in the simulations.

\section{CONCLUSION}

In this paper, we introduced novel reordering algorithms for parallel multi-core network processors that reduce reordering delays without any meaningful additional cost of implementation. The algorithms are scalable and can be implemented over general-purpose processors. We relied on the fact that all packets of a given flow have similar required processing functions, and therefore that we can divide these into an equal number of logical processing phases. We then introduced three frameworks that define the stages at which the NP learns about the number of processing phases: as packets arrive, or as they start being processed, or as they complete processing. In each framework, we introduced a specific reordering algorithm and provided a theoretical model. Finally, we analyzed these algorithms using NP simulations, and found that reordering delays are negligible, both under synthetic traffic and real-life traces. We also showed how a lower variability in the delays of the logical processing phases leads to significant improvements in the performance of our algorithms.

\section{ACKNOWLEDGMENT}

The authors would like to thank Ori Rottenstreich and David Hay for their helpful comments. This work was partly supported by the Hasso Plattner Institute Research School, as well as by the European Research Council Starting Grant No. 210389, the Intel ICRI-CI Center, the Israel Ministry of Science and Technology, the Technion Funds for Security Research, and the J. D. Erteschik Fund for practical research. Support for CAIDA's Internet Traces is provided by the NSF, the US Department of Homeland Security, and CAIDA Members.

\section{REFERENCES}

[1] P. Paulin, F. Karim, and P. Bromley, "Network processors: a perspective on market requirements, processor architectures and embedded S/W tools," in DATE, 2001.

[2] S. Hauger et al., "Packet processing at $100 \mathrm{Gbps}$ and beyond - challenges and perspectives," Photonic Networks, 2009.

[3] M. Peyravian and J. Calvignac, "Fundamental architectural considerations for network processors," Computer Networks, Apr. 2003.

[4] A. Ghodsi, V. Sekar, M. Zaharia, and I. Stoica, "Multi-resource fair queueing for packet processing," in ACM SIGCOMM, 2012.

[5] E. Blanton and M. Allman, "On making TCP more robust to packet reordering," ACM Computer Communication Review, vol. 32, 2002.

[6] V. Paxson, "End-to-end internet packet dynamics," IEEE/ACM Trans. Netw., vol. 7, no. 3, pp. 277-292, Jun. 1999.

[7] Cavium, "Octeon II CN68XX multi-core MIPS64 processors [online]," www.caviumnetworks.com/OCTEON-IICN68XX.html, 2010.

[8] AMCC, "NP7310, 10-Gbps network processor with integrated traffic manager [online]," www.datasheetdir.com/NP7310+ Communications-Processor.

[9] EZChip, "EZChip NP-4 network processor, product brief, 2010. [online]," www.ezchip.com/Images/pdf/NP-4_Short_Brief_online.pdf.

[10] Netronome, "NFP-32xx, the first network flow processor for unified computing. [online]," www.netronome.com/pages/network-flow-processors.

[11] Cisco, "The Cisco QuantumFlow processor. product brief, 2010. [online]," www.cisco.com/en/US/prod/collateral/routers/ps9343/solution overview_c22-448936.pdf.

[12] Juniper, "Junos trio, white paper, 2009. [online]," www.juniper.net/us/en/ local/pdf/whitepapers/2000331-en.pdf.
[13] A. Shpiner, I. Keslassy, and R. Cohen, "Scaling multi-core network processors without the reordering bottleneck, Tech. Report TR14-01, Technion, 2014.” [Online]. Available: webee.technion.ac.il/ isaac/papers. html

[14] N. Weng and T. Wolf, "Pipelining vs. multiprocessors-choosing the right network processor system topology," in ANCHOR, 2004.

[15] W. Shi, M. MacGregor, and P. Gburzynski, "Load balancing for parallel forwarding," IEEE/ACM Trans. Netw., Aug. 2005.

[16] M. Meitinger, R. Ohlendorf, T. Wild, and A. Herkersdorf, "A hardware packet re-sequencer unit for network processors," in ARCS, 2008.

[17] P. He et al., "Balanced locality-aware packet schedule algorithm on multicore network processor," in ICFCC, 2010.

[18] L. Kencl and J.-Y. Le Boudec, "Adaptive load sharing for network processors," in IEEE INFOCOM, 2002.

[19] W. Wu, P. DeMar, and M. Crawford, "Why Does Flow Director Cause Packet Reordering?" ArXiv e-prints, Jun. 2011.

[20] S. Govind, R. Govindarajan, and J. Kuri, "Packet reordering in network processors," in IPDPS, 2007.

[21] B. Wu, Y. Xu, B. Liu, H. Lu, and X. Wang, "An efficient scheduling mechanism with flow-based packet reordering in a high-speed network processor," in HPSR, 2005.

[22] L. Shi et al., "On the extreme parallelism inside next-generation network processors," in IEEE INFOCOM, 2007.

[23] H. Cheng, Y. Jin, Y. Gao, Y. D. Yu, W. Hu, and N. Ansari, "Per-flow resequencing in load-balanced switches by using dynamic mailbox sharing," in $I C C, 2008$.

[24] I. Keslassy, K. Kogan, G. Scalosub, and M. Segal, "Providing performance guarantees in multipass network processors," in IEEE INFOCOM, 2011.

[25] T. Wolf, P. Pappu, and M. A. Franklin, "Predictive scheduling of network processors," Computer Networks, Apr. 2003.

[26] T. Wolf and M. Franklin, "Locality-aware predictive scheduling of network processors," in IEEE ISPASS, 2001, pp. $152-159$.

[27] V. Galtier, K. Mills, Y. Carlinet, S. Bush, and A. Kulkarni, "Predicting and controlling resource usage in a heterogeneous active network," in Active Middleware Services, 2001.

[28] F. Sabrina and S. Jha, "Scheduling resources in programmable and active networks based on adaptive estimations," in IEEE LCN, 2003.

[29] R. Ramaswamy, N. Weng, and T. Wolf, "Considering processing cost in network simulations," in SIGCOMM, 2003.

[30] Ezchip, "Ezchip technologies overview. [online]," www.ezchip.com/ technologies.htm.

[31] K. Karras, T. Wild, and A. Herkersdorf, "A folded pipeline network processor architecture for $100 \mathrm{gbit} / \mathrm{s}$ networks," in ANCS, 2010.

[32] C. Kozanitis, J. Huber, S. Singh, and G. Varghese, "Leaping multiple headers in a single bound: Wire-speed parsing using the kangaroo system," in IEEE INFOCOM, 2010.

[33] O. Rottenstreich, P. Li, I. Horev, I. Keslassy, and S. Kalyanaraman, "The switch reordering contagion: Preventing a few late packets from ruining the whole party," Trans. Computers, to appear, 2013.

[34] L. A. Adamic and B. A. Huberman, "Zipf's law and the internet," Glottometrics, vol. 3, pp. 143-150, 2002.

[35] J. Sobel, "JS hash function, [online]," www.partow.net/programming/ hashfunctions/index.html\#AvailableHashFunctions.

[36] K. Claffy, D. Andersen, and P. Hick, "The CAIDA anonymized 2012 internet traces - (equinix-sanjose/20120119-130000.utc), [online]," www. caida.org/data/passive/passive_2012_dataset.xml.

[37] R. Ramaswamy, N. Weng, and T. Wolf, "Analysis of network processing workloads," in Journal of Systems Architecture, Oct. 2009. 